\title{
Emission wavelength control of ordered arrays of InGaAs/GaAs quantum dots
}

\author{
Irina V. Kulkova ${ }^{a}$, Alexey Lyasota, Clément Jarlov, Bruno Rigal, Alok Rudra, Benjamin \\ Dwir, and Eli Kapon
}

Institute of Physics, Laboratory of Physics of Nanostructures, Ecole Polytechnique Fédérale de Lausanne (EPFL), CH-1015 Lausanne, Switzerland

Growth of InGaAs/GaAs quantum dots (QDs) in inverted pyramids on pre-patterned $\{111\} \mathrm{B}$ GaAs substrates is a versatile technique allowing for precise site and emission energy control. We report on the fabrication of QDs with a wavelength setting within a range of $\sim 100 \mathrm{meV}$ achieved in a single growth step by varying the pyramid size and without compromising the optical quality. Low-temperature micro-photoluminescence spectra of the QD ensembles exhibit low inhomogeneous broadening $(\sim 15 \mathrm{meV})$ and excitonic linewidths as low as $50 \mu \mathrm{eV}$. Moreover, we demonstrate the selective energy tuning of a single QD embedded within an ensemble of QDs spectrally blue-shifted by as much as $40 \mathrm{meV}$, which is of interest for single QD spectroscopy and the fabrication of integrated multi-wavelength single photon sources.

Keywords: A1. Nanostructures; A3. Metalorganic vapor phase epitaxy; A3. Non planar growth; A3. Quantum dots; B2. Semiconducting III-V materials; B2. Indium Gallium Arsenide.

\section{INTRODUCTION}

Semiconductor QDs have gained much attention in the field of photonic quantum technology[1], [2], e.g. for the realization of single-photon sources for integrated quantum circuits[3], [4] and ultra-small lasers[5], owing to their atomic-like electronic and optical properties and compatibility with semiconductor technology. These applications of QDs, especially their integration with other photonic structures such as photonic crystal cavities, often demand fine QD spectral and position control as well as high optical quality. The most common technique for epitaxial QDs fabrication, namely self-assembled Stranski-Krastanov (S-K) growth[6], allows to achieve high density QDs without pre-growth surface preparation, but suffers from large inhomogeneous broadening, from the influence of a wetting layer on carrier capture and recombination processes, and from a large fine structure splitting (FSS) owing to QD asymmetry[7]. The latter is a detrimental issue for applications such as long distance quantum key distribution, where the generation of entangled photons is a key element[8]. Improvements (FSS $\sim 7.6 \mu \mathrm{eV}$ ) in this respect were recently achieved using self-assembled QDs grown on

\footnotetext{
a Electronic mail: irina.kulkova@imec.be. Current affiliation: 1. IMEC, Kapeldreef 75, B-3001 Heverlee, Belgium; 2. Department of Physics and Astronomy, KU Leuven, Celestijnenlaan 200D, B-3001 Heverlee, Belgium
} 
\{111\}A InP substrates under tensile-strain with QDs emitting at $1.267 \mathrm{eV}$ [9]. Alternatively, droplet epitaxy can be employed for growth initiation, with the resulting InGaAs/AlGaAs QDs showing narrow excitonic lines $\sim 40 \mu \mathrm{eV}$ wide[10] and FSS as low as $5.6 \mu \mathrm{eV}[11]$. However, due to the requirements of low-temperature growth, it is difficult to eliminate defect formation due to excess arsenic incorporation and/or sulfur impurities in the latter approach. Another problem associated with S-K and droplet epitaxy arises from the random nucleation positions of the QDs, which limits the feasibility of integration with other photonic elements. This obstacle has been tackled by modifying the growth surface chemical potential, either by using a dielectric mask with apertures[12], [13], by ex- or in-situ etching of nano-cavities[14], [15], or by inducing local strain[16] thereby defining a preferential nucleation point. For example, a small inhomogeneous broadening $(14.4 \mathrm{meV})$ and narrow lines $(>43 \mu \mathrm{eV})$ of QDs were achieved by employing SK growth of InGaAs/GaAs QDs in circular nano-recesses on (100) GaAs substrates[17], and more recently linewidths as low as $6 \mu \mathrm{eV}$ were obtained by SK growth of InAs QDs on a thick GaAs buffer layer grown on a patterned substrate[18]. However, these techniques often result in zero or multi-QD formation around a single intended nucleation center, and hence their site control aspect is compromised.

Control of the emission wavelength of QDs is also desirable or even essential in photonic applications, e.g., for resonant coupling of QD emission to photonic cavities[19]. Tuning of the ensemble energy of S-K QDs by up to $~ 380 \mathrm{meV}$ was demonstrated using post-growth thermal annealing[20], [21] or thermally-induced compositional intermixing via ion implantation[22], but with the drawback of reduced brightness and/or large inhomogeneous broadening. Evidently, simultaneous control of site and emission wavelength while maintaining the high optical quality of the QD systems in terms of linewidths, inhomogeneous broadening and FSS still presents a significant challenge in this field.

The present work is based on the metalorganic vapor phase epitaxy (MOVPE) of InGaAs/GaAs QDs in pyramidal recesses fabricated by electron-beam lithography (EBL) patterning of $\{111\} \mathrm{B}$ GaAs substrates[23]. The formation of these pyramidal QDs is driven by a combination of growth rate anisotropy and nano-capillarity[24]. Major advantages of this approach compared to other techniques include the perfect site control (single QDs always formed at the $20 \mathrm{~nm}$ - sized pyramid tips), the small inhomogeneous broadening of the QD emission spectra (as small as $\sim 6.5$ meV for ensembles, $<2 \mathrm{meV}$ for neutral exciton lines[25]), and small FSS due to the high symmetry of the $\{111\}$ substrates employed $(\sim 7 \mu \mathrm{eV})$. The emission wavelength of pyramidal QDs can be set in a controllable manner by changing the pyramid size or position within specific patterns as a consequence of the introduced changes in growth rates at each pyramid[26]-[28]. A tuning range of $\sim 60 \mathrm{meV}$ has been demonstrated with such an approach, with some degradation in optical quality[28]. Here we demonstrate a tuning range of $\sim 100 \mathrm{meV}$ of uniform arrays of InGaAs/GaAs pyramidal QDs suited for integration in photonic crystal (PhC) membrane cavities[29], while preserving high optical quality (e.g., QD linewidths as narrow as $50 \mu \mathrm{eV}$ ) and perfect site control. In addition, we insert wavelength-shifted pyramidal QDs with regular QD arrays of different wavelengths, in a single growth step. This method gives access to single QD spectroscopy in an "as-grown" environment, and demonstrates the feasibility of deterministic mixed-wavelength QD systems, promising candidates for the realization of multi-wavelength single-photon sources[30]. 


\section{EXPERIMENTAL PROCEDURE}

The InGaAs/GaAs pyramidal QDs were grown by low-pressure (20 mbar) MOVPE in a horizontal Aix200 commercial reactor on semi-insulating vicinal $\{111\} \mathrm{B}$ GaAs substrates tilted by 3 degrees towards the [-211] direction. Prior to growth, the substrates were patterned with $0.5 \times 0.5 \mathrm{~cm}^{2}$ areas containing triangular arrays (pitch $p=450 \mathrm{~nm}$ ) of equilateral triangles of side $s$ using EBL followed by dry etching of the $\mathrm{SiO}_{2}$ mask. Anisotropic wet etching in a $\mathrm{Br}_{2}-$ methanol solution produced inverted tetrahedral pyramids exposing $\{111\}$ A facets at each array site (the details of the sample preparation are described in Ref. [31]). FIG. 1(a) shows scanning electron microscope (SEM) images of such patterned substrates. The pattern geometry and schematics of the QDs grown in the pyramidal pits are presented in FIG. 1(b)

Several arrays of different parameters were fabricated on a single substrate, each one containing pyramids with the same value of $s$, ranging from 200 to $320 \mathrm{~nm}$. The pitch was kept constant at $p=450 \mathrm{~nm}$ corresponding to an areal QD density of $5.7 \times 10^{8} \mathrm{~cm}^{-2}$. MOVPE growth was carried out at $600{ }^{\circ} \mathrm{C}$ (thermocouple temperature) using triethylgallium (TEGa), trimethylgallium (TMGa), trimethylindium (TMIn) and Ultima II $6 \mathrm{~N} 5$ grade arsine $\left(\mathrm{AsH}_{3}\right)$ from Matheson, with nitrogen as carrier gas. The nominal growth rates (as measured on (100) GaAs surfaces) were close to $0.01 \mathrm{~nm} / \mathrm{s}$. Prior to growth, the substrates were deoxidized under $\mathrm{AsH}_{3}$ during $~ 4$ min. The layer sequence consisted of a $2 \mathrm{~nm}$ GaAs buffer layer, a $0.67 \mathrm{~nm}$ $\mathrm{In}_{0.25} \mathrm{Ga}_{0.75} \mathrm{As}$ QD layer, and a $10 \mathrm{~nm}$ GaAs cap layer. TEGa was used in all layers except during the top $8 \mathrm{~nm}$ of the GaAs cap where it was replaced by TMGa to improve the planarization of the final epitaxial surface. The advantage of the use of a TEGa source for the reproducible growth of QDs with reduced linewidths $(\sim 80 \mu \mathrm{eV})$ was reported in more detail in Ref. [31].

After growth, the QDs were investigated using low temperature $\left(\begin{array}{lll}10 & \mathrm{~K}\end{array}\right)$ microphotoluminescence $(\mu$-PL) measurements. The excitation was provided by a continuous-wave Ti:sapphire laser at $720 \mathrm{~nm}$, focused onto the sample using a x50 microscope objective (N.A. $=0.55)$ to form a spot $\sim 1.5 \mu \mathrm{m}$ in diameter. The emitted light was collected through the same objective and analyzed using a single-grating monochromator coupled to a liquid-nitrogen cooled Si CCD, yielding a spectral resolution of about $50 \mu \mathrm{eV}$. For the time-resolved study, the CCD was replaced by an avalanche photodiode (APD) with a time response of $175 \mathrm{ps}$ and the Ti:Sapphire laser was mode-locked to produce 3 ps pulses at $80 \mathrm{MHz}$ repetition rate.

\section{RESULTS AND DISCUSSION}

\section{A. Emission wavelength control}

FIG. 2(a) shows low temperature $\mu$-PL spectra of the QD emission for arrays with $s$ varying from 210 to $320 \mathrm{~nm}$, emitting in the range from 1.38 to $1.28 \mathrm{eV}$, respectively. The QD emission is represented by a series of sharp lines, as exemplified in FIG. 2(b), corresponding to an ensemble of 20 - 40 QDs (the excitation area is assumed to be a few $\mu \mathrm{m}$ in diameter, accounting for carrier diffusion in GaAs). The emission at $1.495 \mathrm{eV}$, attributed to acceptor impurities in GaAs is not representative of the epitaxial layers since it is dominated by the contribution of the GaAs substrate, as observed on substrates of same origin before any epilayer 
is grown. A weak peak at $1.514 \mathrm{eV}$ (not shown here) is due to recombination in bulk GaAs. By fitting the QD ensemble spectra using a Gaussian (see the black dashed curve in FIG.2(b)), the central values of the emission energy and the spectral uniformity (measured as the full width at half maximum (FWHM) of the envelope) as a function of the pyramid size were obtained. The results are plotted in FIG. 3(a) and 3(b). FIG. 3(c) displays the average and minimum values of the linewidth of individual exitonic peaks, which were evaluated using a Lorentzian fit of the lines at the ensemble edge, where the background related to peak superposition is negligible (FIG. 2(b)). Resolution-limited QD linewidths as narrow as $50 \mu \mathrm{eV}$ attest to the high optical quality resulting from the low impurity concentration.

The inhomogeneous broadening of the QD spectrum is due to both the occurrence of pairs of $\mathrm{X}^{\circ}$ and $\mathrm{X}^{-}$(neutral and charged excitons) separated by $\sim 5 \mathrm{meV}$ [32], and to fluctuations in the pyramid side $s$ across the pattern. The latter effect is related to the variation in growth rate at each pyramid due to fluctuation in the area of the $\{111 \mathrm{~A}\}$ growth facets [28]. We note that the emission from the quantum wires formed at the wedges of the pyramids are not observed in the PL spectra.

The QD emission energy increases linearly with increasing size $s$ over nearly the entire size range explored, at a rate of $0.835 \mathrm{meV} / \mathrm{nm}$, covering $\sim 100 \mathrm{meV}$ energy span. This indicates monotonous decrease in QD size and/or a reduced In content with increasing $s$. Such a trend can be explained by an increasing effective growth rate inside the pyramid as the ratio between the \{111\}B and \{111\}A surface areas increases (see FIG. 1(b)), in consistency with previous experiments[26]-[28]. Since the decomposition of the metalorganic precursors takes place mainly inside the pyramids exposing $\{111\}$ A facets, decreasing $s$ while keeping $p$ constant increases the growth rate due to diffusion of precursors into the pyramidal pits. Indeed, the pyramid size $s$ is somewhat larger than the adatom surface diffusion length $(\sim 100 \mathrm{~nm})$ inside the pyramids, which makes the decomposed precursors available for forming the QDs at the tip of the pyramids.

This regular behavior of the QD optical properties with decreasing $s$ exhibits a deviation for the smaller pyramid size studied, $s=200 \mathrm{~nm}$, for which the emission energy increases rather than decreases relative to larger pyramids, and the PL intensity is significantly lower. This might be due to several effects: (i) out-diffusion of $\mathrm{Ga}$ and In adatoms from the smaller pyramidal pit to the surrounding $\{111\} \mathrm{B}$, which reduces the QD thickness and/or the In content; (ii) lower surface curvature evolving at the upper parts of the growing inverted pyramid, which reduces capillarity effects; and (iii) QD positioning closer to the upper surface, which increases nonradiative recombination effects[33].

FIG. 4(a) presents examples of time-resolved $\mu$-PL decay traces measured on exitonic lines within the ensemble spectra. The decay times, extracted using mono-exponential fits, show decreasing values with increasing emission energy (or increasing pyramid size), as presented in FIG. 4(b). This result can be explained by the increase of the exciton oscillatory force, resulting from an increased electron-hole wave function overlap[34], as the QD thickness decreases and its emission energy increases. This observation is in agreement with previous reports[35]-[37] and opposite to other reports[38], [39].

\section{B. Multi-wavelength QD arrays}

Optical characterization of a single QD (sQD) is challenging since ensembles of QDs with high optical quality are typically obtained with high surface density of the QDs. The close 
proximity of dots (typically $<1 \mu \mathrm{m}$ ) necessitates optical isolation of the QDs so that a single dot can be optically excited and analyzed. Often this isolation is achieved by etching a mesa around the $\operatorname{dot}[40]$ or by masking the surrounding QDs with an aperture metallic film[41]. In addition to the complex processing needed, the change in the QD environment may influence the resulting QD spectra due to microresonator effects[42], carrier interaction with sidewall defects[43], induced electrostatic fields, or plasmonic effects [44]. The control over the energy states in a pyramidal QD via the change in the pyramid size $s$ described above offers a new way for the investigation of a SQD in an "as-grown" environment by spectrally shifting its bandgap with respect to its neighboring dots. More specifically, this can be done by producing pyramid patterns in which pyramids of side $s$ ' are inserted within an otherwise regular array of pyramids of side s; subsequent MOVPE of the QDs would then yield spectrally shifted single QDs (pyramid side $s$ ') that are well separated spatially from each other and can be probed using conventional $\mu$-PL spectroscopy.

To demonstrate such structures, the substrate was patterned with $0.5 \times 0.5 \mathrm{~cm}^{2}$ arrays of pyramids with pyramid size $s=320 \mathrm{~nm}$ and pitch $p=450 \mathrm{~nm}$. Single pyramids, spaced by > $4.5 \mu \mathrm{m}$, were "substituted" by smaller pyramids with pyramid size $s$ ' $=175 \mathrm{~nm}, 245 \mathrm{~nm}$ or 285 nm. SEM images and a sketch of the patterned substrate used for this demonstration are shown in FIG. 5. The fabrication process and growth condition were identical to those of the previously discussed experiments. However, it should be noted, that due to fluctuations in the growth conditions from run to run, the exact size and composition of the QDs affect their exact emission wavelengths. In order to provide an adequate reference point in the $\mu$-PL study, uniform arrays of pyramids of side $s$ ' were included in this part of the work.

$\mu$-PL spectra of the grown QD ensembles $(s=320 \mathrm{~nm})$ with embedded, spectrally isolated QDs are shown in FIG. 6(a). The spectra were acquired at low excitation power $\left(2.5 \mathrm{~W} / \mathrm{cm}^{2}\right)$ at $10 \mathrm{~K}$. In order to identify the position of the sQDs, a spatial scanning with a step size of $100 \mathrm{~nm}$ was employed during the $\mu$-PL measurement, seeking the maximum PL intensity at the energy of the sQD emission. The spectra consist of a broad-envelope PL feature from the ensemble of the "matrix" QDs emitting around $1.375 \mathrm{eV}$ (FWHM = $8 \mathrm{meV}$, typical of such ensembles of QDs) and several red-shifted sharp peaks corresponding to the isolated QDs. Magnified views of the spectra of the single QDs for pyramid size $s^{\prime}=175,245$ and $285 \mathrm{~nm}$ are shown in FIG. 6(b)-(d). The two main lines are attributed to the transitions of the negatively charged $\mathrm{X}^{-}$and neutral $\mathrm{X}^{0}$ excitons separated by approximately $5 \mathrm{meV}$, which is a characteristic value for this type of QDs at low excitation power[32]. The near-resolution-limited linewidths $(70-90 \mu \mathrm{eV})$ of the $\mathrm{X}^{-}$lines indicate a good optical quality of the QDs.

The dependence of the QD emission wavelength on the pyramid side $s$ observed for the uniform arrays suggests that also for the mixed-size arrays the wavelength shifts are related to the change in pyramid size. FIG. 7 compares the emission energy of the spectrally isolated QDs (triangles) and the emission energy of QDs in uniform arrays (circles) as a function of pyramid size. Evidently, the red shift for the isolated QDs with decreasing side length is smaller than that for the uniform arrays of QDs of the same pyramid sizes. This indicates that the increase in QD thickness and/or the increase in its In content is reduced for the inserted pyramids as compared to the uniform arrays. The observed effect is consistent with a competition between the inserted pyramid $(s ')$ and the surrounding pyramids $(s)$ on the feeding by the growth precursors, yielding the observed (relative) blue shift in the inserted-pyramid QD emission wavelengths. Yet, the persisting effect of local variations in growth rates is significant, yielding $>40 \mathrm{meV}$ emission wavelength tunability in this case. 


\section{CONCLUSION}

In summary, we have investigated the growth and optical properties of site-controlled InGaAs/GaAs QDs grown in inverted pyramids on $\{111\} \mathrm{B}$ GaAs with respect to the pyramid size as a control parameter for designing the QD emission energy. The study shows linear dependence of the emission energy versus pyramid size in the range from 1.38 to $1.28 \mathrm{eV}$ for pyramid size varying from 320 to $210 \mathrm{~nm}$ (pitch $=450 \mathrm{~nm}$ ). The systematic variation in PL energy indicates controlled variations in the QD size and/or In content within this range of pyramid size variation. These results are consistent with the influence of the pattern geometry on the mass transport and precursor decomposition effects taking place during MOVPE, resulting in the growth rate enhancement in smaller pyramids. $\mu$-PL measurements show resolution-limited QD linewidths as narrow as $50 \mu \mathrm{eV}$ attesting to the high optical quality of the QDs throughout nearly the entire range of controlled energy change $(100 \mathrm{meV})$. The study also shows a systematic change in the PL lifetime for QDs of different size, which reflects the modification of the confinement potential. Moreover, we have demonstrated the tuning of the energy of a single site-controlled QD within a QD ensemble (> $40 \mathrm{meV}$ energy span) by proper design of the pyramid size. This approach allows to isolate single QDs spectrally, without using any postgrowth processing, which is of high interest, e.g., for sQD spectroscopy and for the realization of multi-wavelength single photon sources integrated with broadband PhC circuits[45], [46].

\section{ACKNOWLEDGMENTS}

The authors gratefully acknowledge financial support from the Swiss National Science Foundation through the project "Semiconductor Quantum Nanophotonics based on Ordered Systems of Quantum Wires and Dots".

\section{REFERENCES}

[1] J. L. O’Brien, A. Furusawa, and J. Vučković, "Photonic quantum technologies," Nat. Photonics, vol. 3, no. 12, pp. 687-695, 2010.

[2] S. Tanzilli, A. Martin, F. Kaiser, M. P. de Micheli, O. Alibart, and D. B. Ostrowsky, "On the genesis and evolution of integrated quantum optics," Laser Photonics Rev., vol. 6, no. 1, pp. 115-143, 2012.

[3] D. Englund, A. Faraon, B. Zhang, Y. Yamamoto, and J. Vucković, "Generation and transfer of single photons on a photonic crystal chip.," Opt. Express, vol. 15, no. 9, pp. 5550-5558, 2007. [4] P. Lodahl and S. Stobbe, "Solid-state quantum optics with quantum dots in photonic nanostructures," Nanophotonics, vol. 2, no. 1, pp. 39-55, 2013.

[5] S. Strauf et al., "Self-tuned quantum dot gain in photonic crystal lasers," Phys. Rev. Lett., vol. 96, no. 12, pp. 25-28, 2006.

[6] L. Goldstein, F. Glas, J. Y. Marzin, M. N. Charasse, and G. Le Roux, "Growth by molecular beam epitaxy and characterization of InAs/GaAs strained-layer superlattices," Appl. Phys. Lett., vol. 47, no. 10, pp. 1099-1101, 1985.

[7] A. Schliwa, M. Winkelnkemper, and D. Bimberg, "Impact of size, shape, and composition on piezoelectric effects and electronic properties of $\operatorname{In}(\mathrm{Ga}) \mathrm{As} / \mathrm{GaAs}$ quantum dots," Phys. Rev. B, vol. 76, no. 20, pp. 1-17, 2007. 
[8] H. J. Kimble, “The quantum internet," Nature, vol. 453, no. 7198, pp. 1023-1030, 2008.

[9] C. D. Yerino et al., "Strain-driven growth of GaAs(111) quantum dots with low fine structure splitting," Appl. Phys. Lett., vol. 105, no. 25, p. 251901, 2014.

[10] E. Stock et al., "Single-photon emission from InGaAs quantum dots grown on (111) GaAs," Appl. Phys. Lett., vol. 96, no. 9, p. 093112, 2010.

[11] J. Treu et al., "Substrate orientation dependent fine structure splitting of symmetric In(Ga)As/GaAs quantum dots," Appl. Phys. Lett., vol. 101, no. 2, p. 022102, 2012.

[12] J. Tommila et al., "Nanoimprint lithography patterned GaAs templates for site-controlled InAs quantum dots," J. Cryst. Growth, vol. 323, pp. 183-186, 2011.

[13] J. Herranz, L. Wewior, B. Alén, D. Fuster, L. González, and Y. González, "Role of regrowth interface preparation process for spectral line-width reduction of single InAs sitecontrolled quantum dots," Nanotechnology, vol. 26, no. 19, p. 195301, 2015.

[14] C. Schneider et al., "Single site-controlled In(Ga)As/GaAs quantum dots: growth, properties and device integration," Nanotechnology, vol. 20, no. 43, p. 434012, 2009.

[15] S. Faure, M. Nishioka, S. Ishida, D. Guimard, and Y. Arakawa, "Metal Organic Chemical Vapor Deposition Growth of High Spectral Quality Site-Controlled InAs Quantum Dots Using In situ Patterning," Appl. Phys. Express, vol. 4, no. 11, p. 112001, 2011.

[16] S. Maier et al., "Bright single photon source based on self-aligned quantum dot-cavity systems," Opt. Express, vol. 22, no. 7, pp. 8136-42, 2014.

[17] A. Huggenberger et al., "Narrow spectral linewidth from single site-controlled In(Ga)As quantum dots with high uniformity," Appl. Phys. Lett., vol. 98, no. 13, pp. 15-18, 2011.

[18] M. K. Yakes et al., "Leveraging Crystal Anisotropy for Deterministic Growth of InAs Quantum Dots with Narrow Optical Linewidths," Nano Lett., vol. 13, no. 10, pp. 4870-4875, Oct. 2013.

[19] K. Hennessy et al., "Quantum nature of a strongly-coupled single quantum dot-cavity system," Nature, vol. 445, no. 7130, pp. 896-899, 2007.

[20] T. Yang, J. Tatebayashi, K. Aoki, M. Nishioka, and Y. Arakawa, "Effects of rapid thermal annealing on the emission properties of highly uniform self-assembled InAs/GaAs quantum dots emitting at 1.3 um," Appl. Phys. Lett., vol. 90, no. 2007, pp. 242-244, 2007.

[21] Y. Hino, N. Ozaki, S. Ohkouchi, N. Ikeda, and Y. Sugimoto, "Growth of InAs/GaAs quantum dots with central emission wavelength of $1.05 \mu \mathrm{m}$ using In-flush technique for broadband near-infrared light source," J. Cryst. Growth, vol. 378, pp. 501-505, 2013.

[22] Y. L. Ji, W. Lu, G. B. Chen, X. S. Chen, and Q. Wang, "InAs/GaAs quantum dot intermixing induced by proton implantation," J. Appl. Phys., vol. 93, no. 2, pp. 1208-1211, 2003.

[23] E. Pelucchi, M. Baier, Y. Ducommun, S. Watanabe, and E. Kapon, "High-quality InxGa1-xAs/A10.30Ga0.70As quantum dots grown in inverted pyramids," Phys. Status Solidi B Basic Res., vol. 238, no. 2, pp. 233-236, 2003.

[24] G. Biasiol and E. Kapon, "Mechanism of self-limiting epitaxial growth on nonplanar substrates," J. Cryst. Growth, vol. 201-202, pp. 62-66, May 1999.

[25] A. Mohan et al., "Record-low inhomogeneous broadening of site-controlled quantum dots for nanophotonics," Small, vol. 6, no. 12, pp. 1268-1272, 2010.

[26] E. Pelucchi, S. Watanabe, K. Leifer, B. Dwir, and E. Kapon, "Site-controlled quantum dots grown in inverted pyramids for photonic crystal applications," Phys. E-Low-Dimens. Syst. Nanostructures, vol. 23, no. 3-4, pp. 476-481, 2004.

[27] E. Pelucchi et al., "Mechanisms of quantum dot energy engineering by metalorganic vapor phase epitaxy on patterned nonplanar substrates," Nano Lett., vol. 7, pp. 1282-1285, 2007. [28] M. Felici, P. Gallo, A. Mohan, B. Dwir, A. Rudra, and E. Kapon, "Site-controlled InGaAs quantum dots with tunable emission energy," Small, vol. 5, no. 8, pp. 938-943, 2009. 
[29] V. S. C. Manga Rao and S. Hughes, "Single quantum-dot Purcell factor and $\beta$ factor in a photonic crystal waveguide," Phys. Rev. B - Condens. Matter Mater. Phys., vol. 75, no. December 2006, pp. 1-7, 2007.

[30] M. J. Collins et al., "Integrated spatial multiplexing of heralded single-photon sources," Nat. Commun., vol. 4, p. 2582, Oct. 2013.

[31] B. Rigal et al., "Site-controlled InGaAs/GaAs pyramidal quantum dots grown by MOVPE on patterned substrates using triethylgallium," J. Cryst. Growth, vol. 414, pp. 187-191, 2015.

[32] C. Jarlov, P. Gallo, M. Calic, B. Dwir, A. Rudra, and E. Kapon, "Bound and anti-bound biexciton in site-controlled pyramidal GaInAs/GaAs quantum dots," Appl. Phys. Lett., vol. 101, no. 19, p. 191101, 2012.

[33] C. F. Wang et al., "Optical properties of single InAs quantum dots in close proximity to surfaces," Appl. Phys. Lett., vol. 85, no. 16, pp. 3423-3425, 2004.

[34] Y. C. Zhang, A. Pancholi, and V. G. Stoleru, "Size-dependent radiative lifetime in vertically stacked (In,Ga)As quantum dot structures," Appl. Phys. Lett., vol. 90, no. 18, p. 183104, Apr. 2007.

[35] M. Gurioli, S. Sanguinetti, and M. Henini, "Dynamic quantum-confined stark effect in self-assembled InAs quantum dots," Appl. Phys. Lett., vol. 78, no. 7, pp. 931-933, Feb. 2001.

[36] Z. Xu, Y. Zhang, J. M. Hvam, J. Xu, X. Chen, and W. Lu, "Carrier dynamics in submonolayer InGaAs/GaAs quantum dots," Appl. Phys. Lett., vol. 89, no. 1, p. 013113, Jul. 2006.

[37] K. JongSu et al., "Size Dependence of the Photoluminescence Decay Time Ilin UnstrainedGaAs Quantum Dots," J. Korean Phys. Soc., vol. 55, no. 31, p. 1051, Sep. 2009. [38] J. Bellessa, V. Voliotis, R. Grousson, X. L. Wang, M. Ogura, and H. Matsuhata, "Quantum-size effects on radiative lifetimes and relaxation of excitons in semiconductor nanostructures," Phys. Rev. B, vol. 58, no. 15, pp. 9933-9940, Oct. 1998.

[39] S. Stobbe, J. Johansen, P. T. Kristensen, J. M. Hvam, and P. Lodahl, "Frequency dependence of the radiative decay rate of excitons in self-assembled quantum dots: Experiment and theory," Phys. Rev. B, vol. 80, no. 15, p. 155307, Oct. 2009.

[40] J. Y. Marzin, J. M. Gérard, A. Izraël, D. Barrier, and G. Bastard, "Photoluminescence of single InAs quantum dots obtained by self-organized growth on GaAs," Phys. Rev. Lett., vol. 73, no. 5, pp. 716-719, 1994.

[41] D. Gammon, E. Snow, B. Shanabrook, D. Katzer, and D. Park, "Fine structure splitting in the optical spectra of single GaAs quantum dots," Phys. Rev. Lett., vol. 76, no. 16, pp. 30053008, 1996.

[42] J. M. Gérard et al., "Quantum boxes as active probes for photonic microstructures: The pillar microcavity case," Appl. Phys. Lett., vol. 69, no. 4, p. 449, 1996.

[43] A. Fiore, J. X. Chen, and M. Ilegems, "Scaling quantum-dot light-emitting diodes to submicrometer sizes," Appl Phys Lett, vol. 81, no. 10, pp. 1756-1758, 2002.

[44] D. Press et al., "Ultrafast optical spin echo in a single quantum dot," Nat. Photonics, vol. 4, no. 6, pp. 367-370, 2010.

[45] P. Gallo et al., "Integration of site-controlled pyramidal quantum dots and photonic crystal membrane cavities," Appl. Phys. Lett., vol. 92, p. 263101, 2008.

[46] C. P. Dietrich, A. Fiore, M. Thompson, M. Kamp, and S. Höfling, "GaAs integrated quantum photonics: Towards dense and fully-functional quantum photonic integrated circuits," ArXiv Prepr. ArXiv, vol. 1601.06956, pp. 1-23, 2016. 


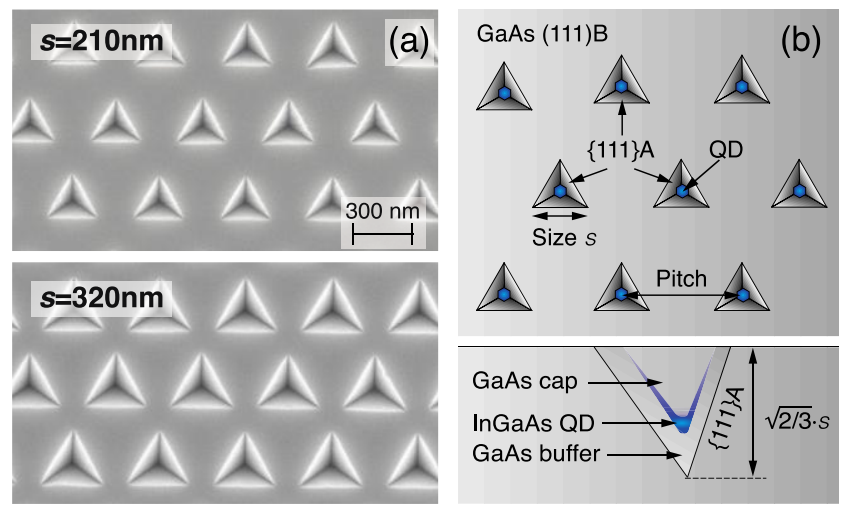

FIG.1. (a) Top view SEM images of arrays of etched pyramids with a pitch $p=450 \mathrm{~nm}$ and a pyramid side length $s=210$ and $320 \mathrm{~nm}$. (b) Schematic top views of a pyramidal QD array and a cross-section of a buried QD after MOVPE growth. The different $\{111\} \mathrm{A}$ and $\{111\} \mathrm{B}$ crystalline surfaces are shown. 

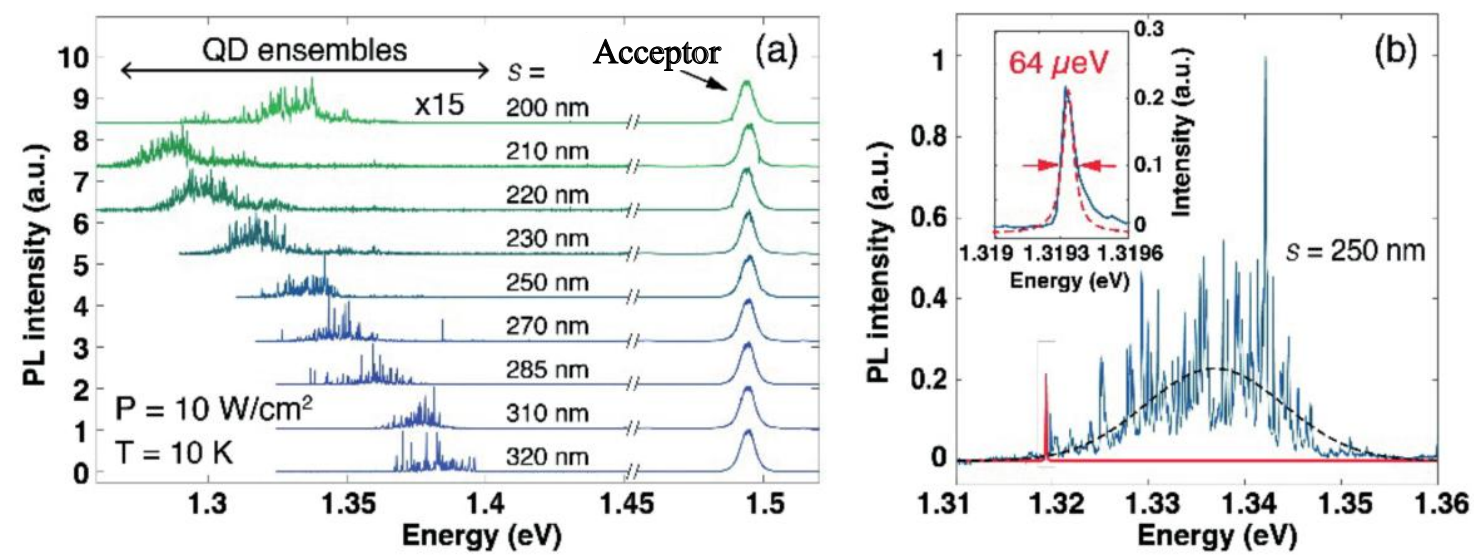

FIG. 2. (a) Low-temperature (10 K) $\mu$-PL spectra of the QD ensembles grown in pyramids with side sizes varying from $s=200$ to $320 \mathrm{~nm}(p=450 \mathrm{~nm})$ measured at $10 \mathrm{~W} / \mathrm{cm} 2$ excitation power. (b) A detailed $\mu$-PL spectrum of a QD ensemble $(s=250 \mathrm{~nm})$. Inset: a magnified view of an individual excitonic line. Red and black dashed lines show the fit used for the extraction of the excitonic line and the inhomogeneous widths (FWHMs), respectively. 


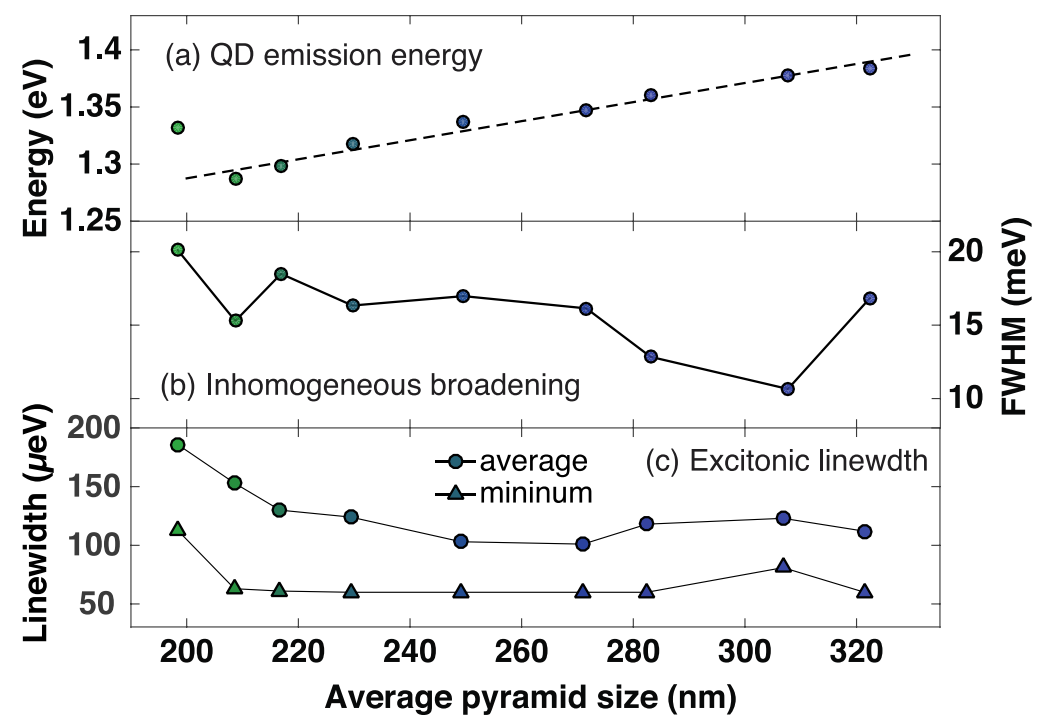

FIG. 3. (a) Emission energy, (b) FWHM of the emission energy distribution of the QD ensembles and (c) Average and minimum linewidth of individual excitonic lines as a function of pyramid size $s$, corresponding to the spectra in Fig. 2. 

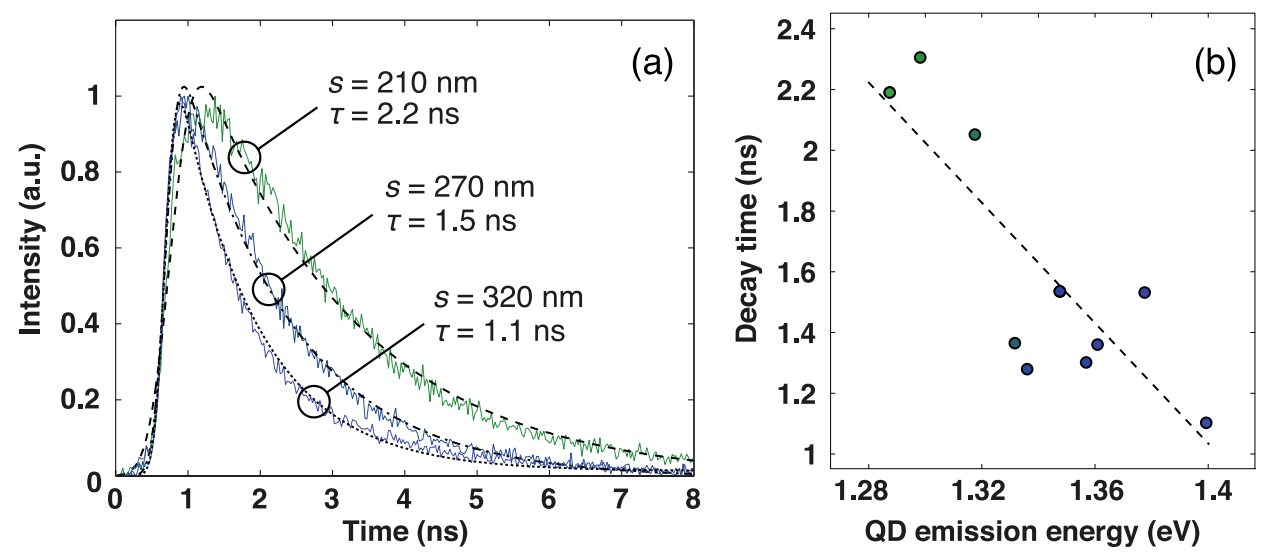

FIG. 4. (a) Examples of time-resolved $\mu$-PL decay traces and (b) correlation between the decay time and the emission energy of the QDs. 

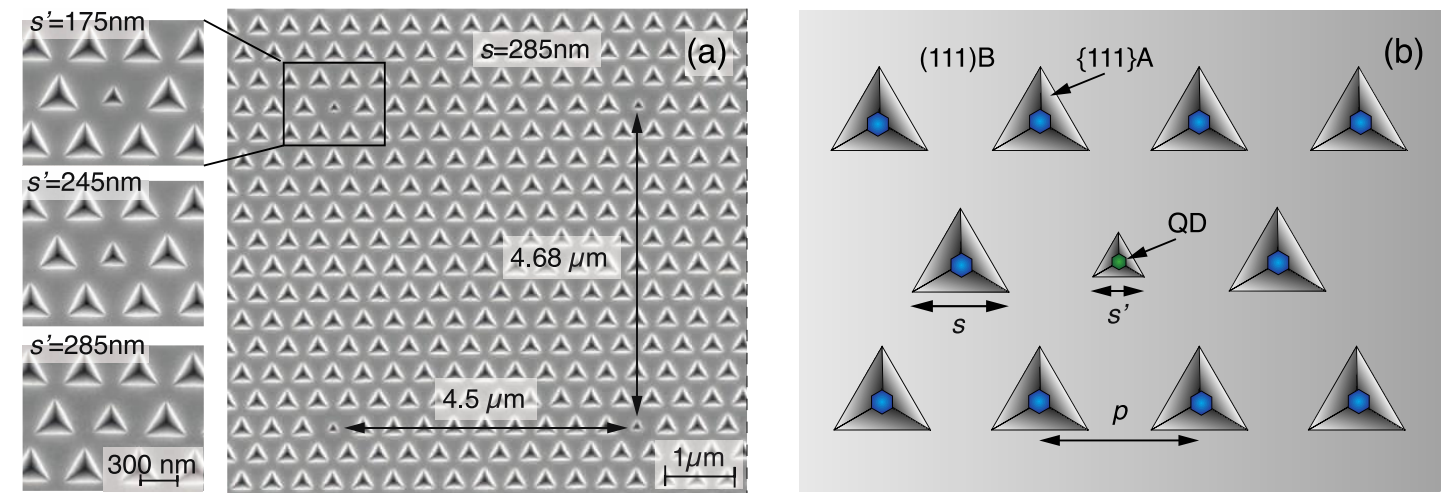

FIG. 5. (a) SEM images of the QD pyramidal arrays consisting of smaller pyramids with $s^{\prime}=175 \mathrm{~nm}, 245 \mathrm{~nm}$ and $285 \mathrm{~nm}$ embedded in a matrix of larger pyramids with $s=320 \mathrm{~nm}(\mathrm{p}=450 \mathrm{~nm})$. (b) Schematic of the pattern used for "spectrally isolated" QDs. 

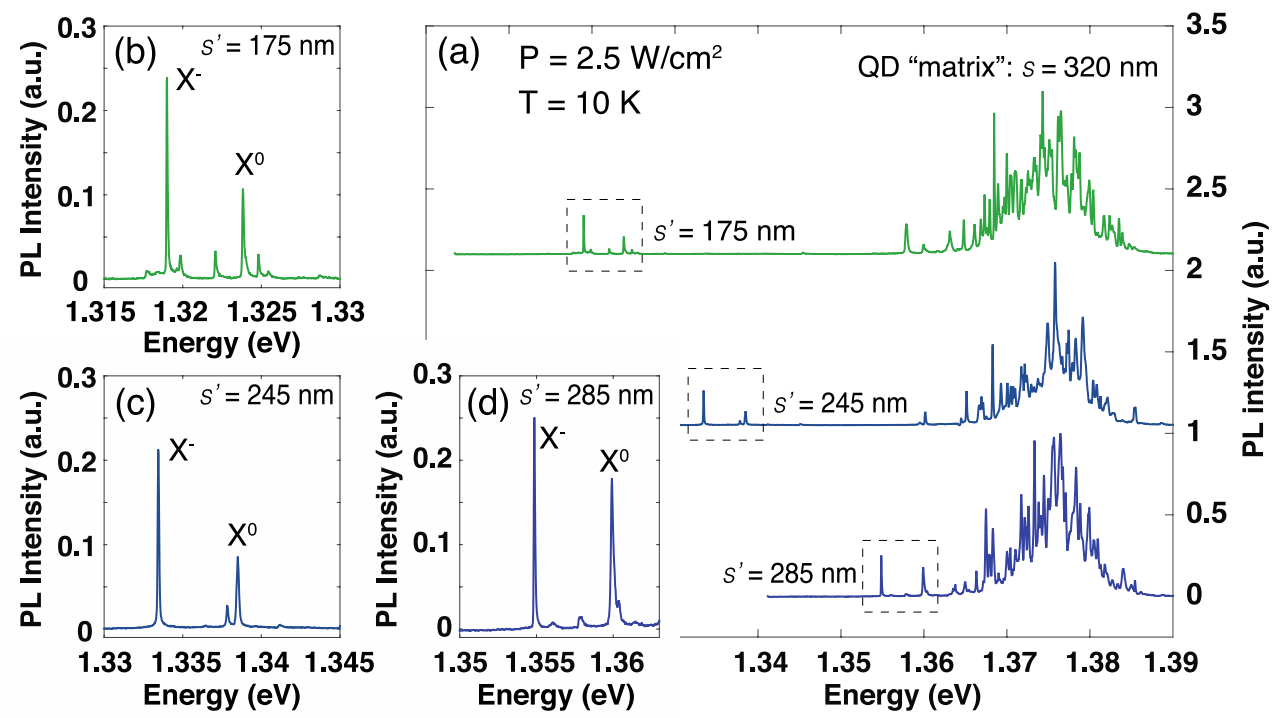

FIG. 6. (a) $\mu$-PL spectra of QD arrays with the pyramid size $s=320 \mathrm{~nm}$ which include spectrally isolated QDs with variable pyramid size $s^{\prime}=175,245$ and $285 \mathrm{~nm}$. (b)-(d) Zoomed-in spectra in the vicinity of the isolated QD lines, for $s^{\prime}=175,245$ and $285 \mathrm{~nm}$, respectively. 


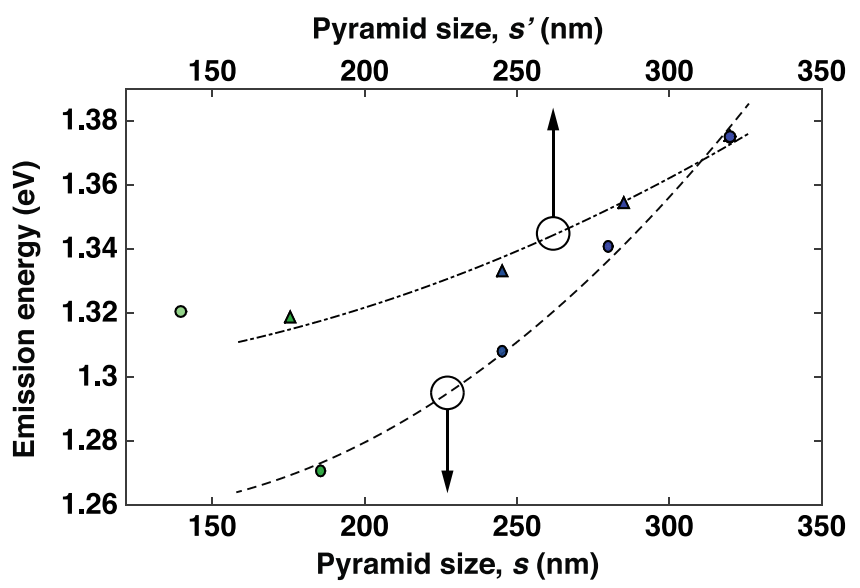

FIG. 7. QD emission energy as a function of pyramid size $s$ for uniform arrays (circles) and arrays consisting spectrally isolated QDs with pyramid size $s^{\prime}$ (triangles) with QD matrices with $s=320 \mathrm{~nm}$. In both cases the pitch is the same, $p=450 \mathrm{~nm}$. 